\title{
Auditory P3a and P3b neural generators in schizophrenia: An adaptive sLORETA P300 localization approach
}

\author{
Alejandro Bachiller ${ }^{1}$, Sergio Romero ${ }^{2,3}$, Vicente Molina, ${ }^{4,5}$, Joan F. Alonso ${ }^{2,3}$, Miguel A. \\ Mañanas $^{2,3}$, Jesús Poza ${ }^{1,5,6}$ and Roberto Hornero ${ }^{1,6}$ \\ ${ }^{1}$ Biomedical Engineering Group, E.T.S. Ingenieros de Telecomunicación, Universidad de \\ Valladolid, 47011 Valladolid, Spain. \{alejandro.bachiller@uva.es; jesus.poza@tel.uva.es; \\ roberto.hornero@tel.uva.es\} \\ 2 Department of Automatic Control (ESAII), Biomedical Engineering Research Center \\ (CREB), Universitat Politècnica de Catalunya (UPC), 08028 Barcelona, Spain. \\ \{sergio.romero-lafuente@upc.edu; joan.francesc.alonso@upc.edu; miguel.angel.mananas@upc.edu\} \\ ${ }^{3}$ CIBER de Bioingeniería, Biomateriales y Nanomedicina (CIBER-BBN) \\ ${ }^{4}$ Psychiatry Department, Hospital Clínico Universitario, Facultad de Medicina, \\ Universidad de Valladolid, 47005 Valladolid, Spain \{vmolina@med.uva.es\} \\ ${ }^{5}$ INCYL, Instituto de Neurociencias de Castilla y León, Universidad de Salamanca, \\ 37007 Salamanca, Spain \\ ${ }^{6}$ IMUVA, Instituto de Investigación en Matemáticas, Universidad de Valladolid, 47011 \\ Valladolid, Spain
}

Corresponding author. Alejandro Bachiller, Biomedical Engineering Group, E.T.S. Ingenieros de Telecomunicación, Universidad de Valladolid, 47011 Valladolid, Spain

Tel: +34 983423000 ext. 5589; fax: +34 983423667; Email address: alejandro.bachiller@uva.es

\begin{abstract}
The present study investigates the neural substrates underlying cognitive processing in schizophrenia (Sz) patients. To this end, an auditory 3-stimulus oddball paradigm was used to identify P3a and P3b components, elicited by rare-distractor and rare-target tones, respectively. Event-related potentials (ERP) were recorded from $31 \mathrm{Sz}$ patients and 38 healthy controls. The P3a and P3b brain-source generators were identified by time-averaging of low-resolution brain electromagnetic tomography (LORETA) current density images. In contrast with the commonly used fixed window of interest (WOI), we proposed to apply an adaptive WOI, which takes into account subjects' P300 latency variability. Our results showed different P3a and P3b source activation patterns in both groups. P3b sources included frontal, parietal and limbic lobes, whereas P3a response generators were localized over bilateral frontal and superior temporal regions. These areas have been related to the discrimination of auditory stimulus and to the inhibition (P3a) or the initiation (P3b) of motor response in a cognitive task. In addition, differences in source localization between Sz and control groups were observed. Sz patients showed lower P3b source activity in bilateral frontal structures and the cingulate. P3a generators were less widespread for Sz patients than for controls in right superior, medial and middle frontal gyrus. Our findings suggest that target and distractor processing involves distinct attentional subsystems, both being altered in Sz. Hence, the study of neuroelectric brain information can provide further insights to understand cognitive processes and underlying mechanisms in Sz.
\end{abstract}

\section{Keywords}

Schizophrenia; LORETA; source localization; event-related potentials; window of interest; P3a and P3b

\section{Introduction}


Alterations in cognitive processing in schizophrenia (Sz) have long been assessed using electroencephalographic (EEG) recordings (Roach and Mathalon, 2008). In particular, it is usual to obtain the event-related potentials (ERP) as the average of EEG epochs time-locked to repeated external stimulus or events. Reduced P300 amplitude during an auditory oddball paradigm is one of the most consistent findings in schizophrenia (Sz) (Bramon et al., 2004); however, the neural bases of this amplitude reduction are incompletely understood. In this regard, the analyses focused on the localization of neural generators can contribute to elucidate possible sources of altered information processing in Sz (Mulert et al., 2004).

The oddball paradigm is a common experimental design used in ERP analyses to obtain the P300 wave. The 3-stimulus variant of the auditory-oddball paradigm is characterized by infrequent-distractor stimuli interspersed randomly into a sequence of frequent-standard and rare-target. This paradigm allows the examination of cognitive processing as response to both relevant and irrelevant stimuli (Polich, 2007). The resulting P300 wave includes two components: the P3a, evoked by distractor stimuli for which no subject-response is expected; and the P3b, elicited by target stimuli for which the subject is instructed to respond. The neural processing as a response to auditory distractor tones has been related to bottom-up attentional mechanisms; hence, P3a may be generated whether sufficient attentional focus is engaged. In contrast, P3b seems to be related to conscious top-down target processing, likely contributing to processing the stimulus information and performing cognitive response (Polich, 2007; Strobel et al., 2008). In previous reports, we found a blunted ERP modulation in Sz as response to both target (Bachiller et al., 2014) and distractor (Bachiller et al., 2015b) tones during an oddball paradigm. Thus, the analysis of the differences in neural pattern generators between Sz patients and healthy controls becomes an interesting research topic to clarify the neural substrate of reduced P300 amplitude in Sz.

Source imaging techniques may help to detect neural generators that contribute to the scalp recorded ERPs, resulting in an acceptable compromise between spatial and temporal resolutions. The inverse solution (i.e. the computation of 3-D intracerebral images of electric neuronal activity based on scalp-recorded EEG) would provide useful information on the time course and localization of brain functions (Pascual-Marqui, 2002). There is no unique solution to the inverse problem; nevertheless, the low-resolution brain electromagnetic tomography (LORETA) is one of the most reliable methods for localizing ERP electrical activity and it is associated with relatively low error rates (Pascual-Marqui, 2002; Jung et al., 2012). LORETA has been widely used for source localization in psychiatric disorders, such as $\mathrm{Sz}$ or depression (Kawasaki et al., 2004; Mientus et al., 2002). Moreover, auditory P3a and P3b source localization has been previously addressed in healthy controls using LORETA and functional magnetic resonance imaging (fMRI). P3a generators are localized in anterior cingulate, frontal 
area and parietal cortices (Polich, 2007; Strobel et al., 2008; Volpe et al., 2007), whereas P3b sources include a more distributed network, involving superior and medial temporal, posterior parietal, hippocampal, cingulate and frontal structures (Polich, 2007; Strobel et al., 2008; Volpe et al., 2007; Wronka et al., 2012).

It is noteworthy that the previous LORETA findings are influenced by one important technical shortcoming: although ERP analyses show a considerable inter-subject variability of P300 latency (Campanella et al., 1999), LORETA source imaging studies commonly used a large fixed post-stimulus window of interest (WOI), like [250 500] ms (Higuchi et al., 2008; Kawasaki et al., 2007; Sumiyoshi et al., 2006), [280 450] ms (Wang et al., 2003, 2010), [240 420] ms (Pae et al., 2003), [227 383] ms (Volpe et al., 2007), or [400 700] ms (Wronka et al., 2012).

In this study we proposed a new LORETA approach based on P300 wave ('P300 latency adaptive WOI') to properly localize P300 brain-source generators in each subject. To the best of our knowledge, this is the first study that analyzes the sources of both P3a and P3b in Sz using LORETA. Hence, this research is aimed at: (i) analyzing the performance of the adaptive WOI method in comparison to conventional fixed WOI analysis; and (ii) applying the adaptive WOI method to analyze the differences of auditory P3a and P3b underlying cortical sources between Sz patients and healthy controls.

\section{Materials and methods}

\subsection{Subjects}

Thirty-one patients with paranoid Sz (Diagnostic and Statistical Manual of Mental Disorders, Fourth Edition, DSM-IV-TR, criteria) and 38 healthy controls were recruited. Sz group was composed by 20 chronic stably treated patients, 7 fist-episode patients and 4 patients who had dropped their medications for a period longer than 6 months. Chronic patients were previously treated with atypical antipsychotics. First-episode patients have not been received previous antipsychotic treatment, except for a brief time interval of less than 72 hours prior to EEG acquisition. No medications were administered to the patients during the 12 hours preceding the EEG recording. Controls were initially assessed by a semi-structured psychiatric interview to discard major psychiatric antecedents and treatments. Detailed description of treatments and doses, as well as exclusion criteria are detailed in previous reports (Bachiller et al., 2015a; Bachiller et al., 2015b). Symptoms were scored using the Positive and Negative Syndrome Scale (PANSS) (Kay et al., 1987). Socio-demographic and clinical characteristics for both groups are shown in Table 1.

The research boards of the Hospitals of Valladolid and Salamanca (Spain) endorsed the study according to The Code of Ethics of the World Medical Association (Declaration of 
Helsinki). Moreover, written informed consent was obtained from patients, their caregivers and healthy volunteers.

\subsection{EEG recording procedure}

EEG recordings were performed while subjects underwent a 3-stimulus auditory-oddball paradigm. Participants heard a random series of 600 binaural tones $(90 \mathrm{~dB} ; 50 \mathrm{~ms}$ duration; 5 ms rise and fall-time) consisting on standard (2000 Hz tone), distractor (1000 Hz tone) and target tones (500 $\mathrm{Hz}$ tone) with probabilities of $0.6,0.2$ and 0.2, respectively (Bachiller et al., 2015a).

For each subject, 13 minutes of EEG activity and stimulus markers were continuously recorded using a 17-channel (Fp1, Fp2, F3, F4, C3, C4, P3, P4, O1, O2, F7, F8, T5, T6, Fz, Pz and $\mathrm{Cz}$ ) EEG system (BrainVision ${ }^{\circledR}$, Brain Products $\mathrm{GmbH}$; Munich, Germany). Electrodes were placed according to the revised 10/20 International System. Participants were relaxed and with their eyes closed. EEG data were recorded at a sampling frequency of $250 \mathrm{~Hz}$ and referenced over $\mathrm{Cz}$ electrode. Electrode impedance was always kept under $5 \mathrm{k} \Omega$.

Each EEG recording was off-line re-referenced to the common average (Bledowski et al., 2004) and digitally filtered using a [0.5 40] Hz finite impulse response filter. Then, a three-step artifact rejection method was applied. Firstly, an independent component analysis was performed to decompose EEG signals (Delorme and Makeig, 2004). After a visual inspection of the scalp maps and their temporal activation, components related to eyeblinks were discarded. In a second step, continuous EEG data were segmented from $-100 \mathrm{~ms}$ before target stimulus onset to $900 \mathrm{~ms}$ after onset. In a third step, artifacts were automatically rejected using an adaptive thresholding method (Bachiller et al., 2015a).

Finally, the responses to distractor and target tones were baseline-corrected by subtracting the $100 \mathrm{~ms}$ pre-stimulus mean and they were averaged across time-locked trials to obtain ERP data for each channel (Pae et al., 2003).

\subsection{Identification of ERP components}

The P300 wave is an ERP component commonly used to assess the neural underpinnings of cognition (Polich, 2007). P300 amplitude was obtained from ERP data as the most positive voltage between 250 and $550 \mathrm{~ms}$, whereas P300 latency was defined as the time point from stimulus onset at which the peak amplitude is found (Polich, 2007). A P300 latency variability has been found across different subjects, which may be related to inter-subject anatomical variability and differences in cognitive attentional processing (Campanella et al., 1999; Polich and Herbst, 2000). In addition, it has been previously shown that P300 latency varies with the discriminability of relevant stimuli: the more difficult is to identify the stimuli, the greater the latency (Furdea et al., 2009; Sellers and Donchin, 2006; Squires et al., 1977). Indeed, this result 
can be also observed in our database: Fig. 1a shows a large P300 latency variability for healthy controls and Sz patients. An adaptive WOI takes into account changes in peak latency across subjects. Therefore, the selection of the WOI can be critical to characterize neural generators (Kim et al., 2014).

In spite of such latency variability, most of the previous P300 source studies applying LORETA have been carried out using a fixed WOI. Recent researches proposed the use of two different fixed WOIs, one for Sz patients and other for healthy controls (Jung et al., 2012; Kim et al., 2013, 2014). Nevertheless, this methodology does not take into account the P300 variability among subjects within each group. In order to overcome this limitation, and therefore to improve $\mathrm{P} 3 \mathrm{a}$ and $\mathrm{P} 3 \mathrm{~b}$ detection and source localization procedures, we propose an algorithm for identifying a subject-specific WOI based on N200-P300 waves. The N200 wave is a negative voltage observed as a response to a visual or an auditory task. It reflects the detection of some type of mismatch between stimulus features (Folstein and Van Petten, 2008).

The proposed subject-specific algorithm was divided into two steps. Firstly, the ERP wave was filtered by a moving average of 9 samples $(36 \mathrm{~ms})$, a smoothing procedure commonly used in P300 detection (Furdea et al., 2009; Sellers and Donchin, 2006). Then, a subject-adaptive WOI threshold was established using the $75 \%$ rise time from N200 amplitude to P300 amplitude (i.e. the time to reach $75 \%$ of the P300 peak amplitude (Mooney and Prather, 2005)).

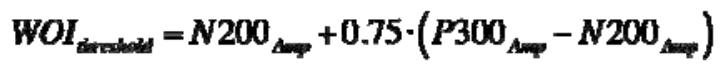

Adaptive WOI interval was obtained as the time points where the ERP amplitude increases up to $W O I_{\text {threshold }}$ before P300 and decreases down to $W_{\text {Ohreshold }}$ after P300. Fig. 1 displays P300 latency values and adaptive WOI limits for all subjects. In addition, it shows how adaptive WOI is better fitted to the P300 component than a fixed WOI.

\subsection{SLORETA}

Bioelectrical ERP neural sources distribution was assessed using standardized LORETA (sLORETA) software, which estimates a particular solution of the non-unique EEG inverse solution (Pascual-Marqui et al., 2002). sLORETA software divides the brain into a total of 6239 cubic voxels with $5 \mathrm{~mm}$ resolution and estimates the source current density (i.e. the sum of electric neuronal activity on each defined voxel) (Pascual-Marqui, 2002). In particular, sLORETA source current density are calculated from scalp-recorded ERP using a realistic head model from Montreal Neurological Institute (MNI) (Mazziotta et al., 2001), in which the 3-D solution space was restricted to only the cortical gray matter (Lancaster et al., 2000). Compared to previous versions of LORETA, sLORETA is superior in temporal resolution and has fewer localization errors (Kim et al., 2014). Thus, sLORETA has been used in several studies to 
investigate brain sources during the generation of ERP components, such as P300 (Jung et al., 2012; Kim et al., 2013, 2014; Sumiyoshi et al., 2009).

In order to consider P300 latency changes across subjects, P3a and P3b source images for each participant were averaged using the time window defined by their subject-adaptive WOI. Fig. 1d shows an example of differences in brain activation patterns for fixed and adaptive WOI.

\subsection{Statistical analysis}

Statistical differences between P3a and P3b sLORETA images were assessed by t-tests, computed for log-transformed sLORETA current density at each voxel (Volpe et al., 2007). Sz patients were compared to healthy controls in order to identify the effect of illness in neural generators.

Within-group differences between P3a and P3b were evaluated using voxel-by-voxel pairedsample $t$-tests, whereas voxel-by-voxel independent $t$-tests were conducted to analyze betweengroup statistics. To correct for multiple comparisons, two nonparametric permutations tests (NPT) based on the theory of randomization were applied: (i) voxel intensity and (ii) cluster size (Nichols and Holmes, 2002). Voxel intensity NPT calculates a critical $t$-value by means of a random sample of all the possible permutations to estimate the distribution of the maximum $t$ statistic. Based on the hypothesis that the activated/deactivated brain region is expected to occur in a cluster of voxels, rather than in isolated voxels, cluster size NPT is a version of voxel intensity test, which allows the calculation of the critical cluster size to be considered significant. Both NPTs provide a corrected $p$-value $\left(p_{c o r}\right)$, which is used to identify statistically significant results.

\section{Results}

Table 1 presents the grand-average ERP amplitude and latency values, as well as the adaptive WOI length and the corresponding aWOI limits for each group. Statistically significant differences between groups (Kruskal-Wallis test, $p<0.05$ ) were only obtained for P3a ( $p=$ $0.0007)$ and $\mathrm{P} 3 \mathrm{~b}(p=0.0036)$ amplitudes. Although a large P300 latency and an adaptive WOI variability across subjects have been observed (Fig. 1), non-statistical differences between groups were obtained.

Based on the observation of normalized grand-averages of auditory P3a and P3b brain sources for Sz patients and healthy controls (Fig. 2), P3a response sources were mainly localized in bilateral frontal (superior, medial and inferior gyrus) and right superior temporal lobes. On the other hand, P3b sources were observed over a large distributed network, including cingulate gyrus, as well as bilateral frontal lobe and parietal lobe (Fig. 2). 
Fig. 3 depicts SLORETA $t$-statistics maps for within-group comparisons between distractor and target stimulus type. Both groups exhibit a greater P3b source current density than P3a response. Statistical differences were located mainly in bilateral limbic lobe and parietal structures (voxel intensity NPT, $p_{c o r}<0.05$ ). Furthermore, a P3b source current density increase was observed for Sz patients mainly in bilateral parietal and limbic lobes, as well as superior and medial frontal regions (Fig. 3, Table 2).

A smaller source current density in Sz patients in comparison with controls was obtained for both components: P3a and P3b. Nevertheless, differences in neural activation patterns depended on stimulus tone. Fig. 4 shows the statistically significant $t$-values distribution for P3a and P3b (cluster size NPT, $p_{c o r}<0.05$ ). In particular, P3b source current density was significantly smaller for Sz patients than for controls in superior, middle and medial frontal gyrus, and cingulate gyrus. On the other hand, reduced P3a in Sz patients was mainly observed in frontal areas (Table 3).

\section{Discussion}

The aim of the present study was to provide further insights into the neural correlates of target (P3b) and distractor (P3a) processing in Sz. A 3-tone auditory-oddball paradigm was used to characterize P3b and P3a brain sources and to compare them between Sz patients and controls.

Firstly, regarding the validity of the proposed P300 latency adaptive WOI algorithm, our research shows how the selection of the WOI influences the sLORETA findings. A wide fixed WOI has been commonly used to solve the problem of P300 latency variability (Kim et al., 2014). However, sLORETA image averaging over a fixed WOI can include several activities strictly non-related to the P300 component. A fixed WOI commonly obtains a smoother source current density that includes a large source activity in medial and superior frontal structures. This frontal activation may be caused by the inclusion of N200 component in the fixed WOI. As shown in Fig. 1d, an adaptive WOI allows focusing on the brain-source generators of P300 component. Consequently, we can conclude that an adaptive WOI may improve P300 source localization procedures.

\subsection{P3a and P3b neural generator patterns}

Our results using an adaptive WOI suggest that common brain areas are activated as a response to target (P3b) and distractor (P3a) tones (Fig. 2), including right superior temporal gyrus and bilateral frontal lobe. This finding is in agreement with previous reports (Bledowski et al., 2004; Strobel et al., 2008; Volpe et al., 2007). Prior research has demonstrated that discrimination between standard and non-standard infrequent stimuli reflects frontal lobe activation, sensible to attentional allocation (Goldstein et al., 2002; Polich, 2007; Volpe et al., 
2007). Our findings also concern regions distinctly activated by distractor and target stimuli. Likewise, differences in P300 brain-source generators are coherent with previous studies in healthy subjects: P3a generators were identified over frontal structures and P3b source activation over a distributed network, including frontal, temporal, limbic and parietal lobes (Anderer et al., 2003; Volpe et al., 2007; Wronka et al., 2012). It is noteworthy that these different patterns may have functional significance. $\mathrm{P} 3 \mathrm{~b}$ is originated as a temporo-parietal mechanism that performs memory and executive control functions (Polich, 2007; Polich and Criado, 2006). On the contrary, P3a reflects the inhibition of a response engaged automatically with the detection of the stimulus deviance (Goldstein et al., 2002). In this sense, frontal lobe activity related to the hippocampus can be associated with the distractor-triggered inhibitory processes (Polich, 2007).

\subsection{Between-group differences}

We found statistically significant differences in the activation of brain sources between patients and controls for both P3a and P3b. We are aware of only one study addressing brain source localization of auditory P3a in Sz (Takahashi et al., 2013). Its results revealed widespread deficits in Sz patients (including frontal, temporal and parietal lobes) with postcentral maximum. Our patients showed a lower P3a source activation in frontal (BA6, BA8, BA9) and cingulate regions (BA24, BA32) (Fig. 4, Table 3). Accordingly, within-group differences (P3b vs. P3a) pointed out the role of these areas in auditory 3-stimulus oddball characterization (Table 2). In this regard, superior and middle frontal (BA6, BA9), cingulate (BA32) and posterior cingulate gyrus (BA23) were commonly involved in auditory P3a generation for healthy controls (Strobel et al., 2008; Volpe et al., 2007). Interestingly, P3a current density reduction for Sz patients in comparison with controls was mainly observed in the right hemisphere of superior, medial and middle frontal cortex (Fig. 4, Table 3). A right lateralization has been associated with the detection of behaviorally relevant or unexpected stimuli (Strobel et al., 2008). Right hemisphere is crucially involved in neural processes underlying adaptive responses to novelty, mainly in perceptual and attention paradigms (Cabeza et al., 2008; Strobel et al., 2008). According to our results, this adaptive function may be hampered in Sz patients. It might hypothetically contribute to aberrant salience in this syndrome through a decreased response to relevance (Kapur, 2003).

In comparison to controls, $\mathrm{P} 3 \mathrm{~b}$ source activation was smaller in Sz patients over superior and medial frontal gyrus (BA10, BA11), orbital frontal gyrus (BA11), bilateral anterior cingulate gyrus (BA33) and cingulate gyrus (BA31, BA32, BA25). Our findings are consistent with previous auditory 2-tone oddball studies focused on P3b source localization in Sz. Sabeti et al. (2011) described a hypoactivation for Sz patients $(n=20)$ in the cingulate area. Kim et al. (2014) showed decreased cingulate (BA31) activation and a negative correlation with negative 
symptoms in the posterior cingulate (BA31) for Sz patients $(n=34)$. Likewise, Mucci et al. (2007) reported a P3b current density reduction in $18 \mathrm{Sz}$ patients compared to 20 controls during a 3-tone oddball paradigm in bilateral frontal and cingulate areas. These studies and our results support the role of cingulate on information processing deficits in Sz. Furthermore, the decreased P3b source activation in Brodmann areas 10 and 11 obtained by Sz patients is consistent with the left middle frontal decrease of current density observed in $16 \mathrm{Sz}$ patients by Higuchi et al. (2008). Interestingly, in spite of the aforementioned decrease of P3b sources in frontal areas for Sz patients, the differences between P3b and P3a were larger in patients than in controls (Fig. 3). This result may indicate an inefficient hyperactivation during the processing of target stimuli. Moreover, our findings showed a left-lateralized P3b increase in comparison with P3a for controls, in contrast to the bilateral temporal increase for Sz patients (Fig. 3). These findings agree with previous auditory oddball reports for healthy controls. A statistically significant P3b increase in left BAs 7 and 19 was previously reported $(n=32)$ (Volpe et al. 2007), as well as a P3b increase in left precuneus (BA19) ( $n=28)$ (Wronka et al., 2012). It is noteworthy that target response involves the activation of associative cortices involved in attentional, perceptual and memory processes (Volpe et al., 2007). In this regard, Corbetta and Shulman (2002) pointed out that left parietal cortex might be involved in assembling associations that link the appropriate stimuli to the response for a given task.

Taken together with previous literature, our findings suggest a relevant role of frontal and cingulate hypoactivation in the deficient response of Sz patients in P300 paradigms (Bramon et al., 2004). Neuropathological (Benes, 2009; Hashimoto et al., 2008) and functional (GuerreroPedraza et al., 2012; Lynall et al., 2010) abnormalities in these regions have been associated with Sz. They might underlie their decreased activation during the task performance. The frontal lobe has been related to the performance of discriminatory tasks (Pae et al., 2003), whereas the cingulate has been assumed to be involved in both the effortful initiation of motor response and the inhibition of motor responses (Liddle et al., 2001). Therefore, deficient capacities for discrimination, inhibition and/or initiation of responses may mediate the role of cingulate and frontal regions in P3b source activity.

\subsection{Limitations and future work}

Some limitations of this research merit further consideration. It would be interesting to evaluate sources of brain activity over a larger database of first-episode patients and relatives. The comparison between chronic and first-episode patients may contribute to characterize the effect of antipsychotic treatment in the neural generators. Moreover, results were limited by the technical constraints associated with sLORETA analysis. However, the effects of these limitations were reduced by using randomization and NPT procedures. Finally, future studies will complement the obtained sLORETA patterns analyzing detailed time-course source 
activation, effective and functional connectivity at the source level, and cross-frequency neural coupling.

\subsection{Conclusions}

In summary, this research supports the use of an adaptive WOI to accurately localize the P300 brain-source generators. Furthermore, we found that cortical source generators during an auditory-oddball task are altered in Sz patients. P3a and P3b source differences between groups were mainly obtained in areas related to stimulus discrimination capacity and motor response tasks. Hence, the study of sLORETA neural current density can improve the pathophysiological characterization of Sz.

\section{References}

Anderer, P., Saletu, B., Semlitsch, H.V., Pascual-Marqui, R.D., 2003. Non-invasive localization of P300 sources in normal aging and age-associated memory impairment. Neurobiol. Aging 24, 463-479.

Bachiller, A., Díez, A., Suazo, V., Domínguez, C., Ayuso, M., Hornero, R., Poza, J., Molina, V., 2014. Decreased spectral entropy modulation in patients with schizophrenia during a P300 task. Eur. Arch. Psychiatry Clin. Neurosci. 264, 533-543.

Bachiller, A., Poza, J., Gómez, C., Molina, V., Suazo, V., Hornero, R., 2015a. A comparative study of event-related coupling patterns during an auditory oddball task in schizophrenia. J. Neural Eng. 12, 016007.

Bachiller, A., Lubeiro, A., Díez, A., Suazo, V., Domínguez, C., Blanco, J.A., Ayuso, M., Hornero, R., Poza, J., Molina, V., 2015b. Decreased entropy modulation of EEG response to novelty and relevance in schizophrenia during a P300 task. Eur. Arch. Psychiatry Clin. Neurosci. 265, 525-535.

Benes, F.M., 2009. Neural circuitry models of schizophrenia: is it dopamine, GABA, glutamate, or something else? Biol. Psychiatry 65, 1003-1005.

Bledowski, C., Prvulovic, D., Hoechstetter, K., Scherg, M., Wibral, M., Goebel, R., Linden, D.E., 2004. Localizing P300 generators in visual target and distractor processing: a combined event-related potential and functional magnetic resonance imaging study. J. Neurosci. 24, 9353-9360.

Bramon, E., Rabe-Hesketh, S., Sham, P., Murray, R.M., Frangou, S., 2004. Meta-analysis of the P300 and P50 waveforms in schizophrenia. Schizophr. Res. 70, 315-329.

Campanella, S., Gomez, C., Rossion, B., Liard, L., Debatisse, D., Dubois, S., Delinte, A., Bruyer, R., Crommelinck, M., Guérit, J.., 1999. Comparison between grand-average and individual analyses: an study. Neurophysiol. Clin. Neurophysiol. 29, 325-338.

Delorme, A., Makeig, S., 2004. EEGLAB: an open source toolbox for analysis of single-trial EEG dynamics including independent component analysis. J. Neurosci. Methods 134, 9-21.

Furdea, A., Halder, S., Krusienski, D.J., Bross, D., Nijboer, F., Birbaumer, N., Kübler, A., 2009. An auditory oddball (P300) spelling system for brain-computer interfaces. Psychophysiology 46, 617-625.

Folstein, J.R., Van Petten, C., 2008. Influence of cognitive control and mismatch on the N2 component of 
the ERP: a review. Psychophysiology 45, 152-170.

Goldstein, A., Spencer, K.M., Donchin, E., 2002. The influence of stimulus deviance and novelty on the P300 and novelty P3. Psychophysiology 39, 781-790.

Guerrero-Pedraza, A., McKenna, P.J., Gomar, J.J., Sarro, S., Salvador, R., Amann, B., Carrion, M.I., Landin-Romero, R., Blanch, J., Pomarol-Clotet, E., 2012. First-episode psychosis is characterized by failure of deactivation but not by hypo-or hyperfrontality. Psychol. Med. 42, 73-84.

Hashimoto, T., Bazmi, H.H., Mirnics, K., Wu, Q., Sampson, A.R., Lewis, D.A., 2008. Conserved regional patterns of GABA-related transcript expression in the neocortex of subjects with schizophrenia. Am. J. Psychiaty. 165, 479-489.

Higuchi, Y., Sumiyoshi, T., Kawasaki, Y., Matsui, M., Arai, H., Kurachi, M., 2008. Electrophysiological basis for the ability of olanzapine to improve verbal memory and functional outcome in patients with schizophrenia: a LORETA analysis of P300. Schizophr. Res. 101, 320-330.

Jung, H.-T., Kim, D.-W., Kim, S., Im, C.-H., Lee, S.-H., 2012. Reduced source activity of event-related potentials for affective facial pictures in schizophrenia patients. Schizophr. Res. 136, 150-159.

Kapur, S., 2003. Psychosis as a State of Aberrant Salience: A Framework Linking Biology, Phenomenology, and Pharmacology in Schizophrenia. Am. J. Psychiatry 160, 13-23.

Kawasaki, T., Tanaka, S., Wang, J., Hokama, H., Hiramatsu, K., 2004. Abnormalities of P300 cortical current density in unmedicated depressed patients revealed by LORETA analysis of event-related potentials. Psychiatry Clin. Neurosci. 58, 68-75.

Kawasaki, Y., Sumiyoshi, T., Higuchi, Y., Ito, T., Takeuchi, M., Kurachi, M., 2007. Voxel-based analysis of P300 electrophysiological topography associated with positive and negative symptoms of schizophrenia. Schizophr. Res. 94, 164-171.

Kay, S.R., Fiszbein, A., Opler, L.A., 1987. The positive and negative syndrome scale (PANSS) for schizophrenia. Schizophr. Bull. 13, 261-276.

Kim, D.-W., Kim, H.-S., Lee, S.-H., Im, C.-H., 2013. Positive and negative symptom scores are correlated with activation in different brain regions during facial emotion perception in schizophrenia patients: A voxel-based sLORETA source activity study. Schizophr. Res. 151, 165-174.

Kim, D.-W., Shim, M., Kim, J.-I., Im, C.-H., Lee, S.-H., 2014. Source activation of P300 correlates with negative symptom severity in patients with schizophrenia. Brain Topogr. 27, 307-317.

Lancaster, J.L., Woldorff, M.G., Parsons, L.M., Liotti, M., Freitas, C.S., Rainey, L., Kochunov, P.V., Nickerson, D., Mikiten, S.A., Fox, P.T., 2000. Automated Talairach Atlas labels for functional brain mapping. Hum. Brain Mapp. 10, 120-131.

Liddle, P.F., Kiehl, K.A., Smith, A.M., 2001. Event-related fMRI study of response inhibition. Hum. Brain Mapp. 12, 100-109.

Lynall, M.-E., Bassett, D.S., Kerwin, R., McKenna, P.J., Kitzbichler, M., Muller, U., Bullmore, E., 2010. Functional connectivity and brain networks in schizophrenia. J. Neurosci. 30, 9477-9487.

Mazziotta, J., Toga, A., Evans, A., Fox, P., Lancaster, J., Zilles, K., et al., 2001. A probabilistic atlas and reference system for the human brain: International Consortium for Brain Mapping (ICBM). Philos.

Trans. R. Soc. B Biol. Sci. 356, 1293-1322.

Mientus, S., Gallinat, J., Wuebben, Y., Pascual-Marqui, R.D., Mulert, C., Frick, K., Dorn, H., Herrmann, 
W.M., Winterer, G., 2002. Cortical hypoactivation during resting EEG in schizophrenics but not in depressives and schizotypal subjects as revealed by low resolution electromagnetic tomography (LORETA). Psychiatry Res. Neuroimaging 116, 95-111.

Mooney, R., Prather, J.F., 2005. The HVC Microcircuit: The synaptic basis for interactions between song motor and vocal plasticity pathways. J. Neurosci. 25, 1952-1964.

Mucci, A., Galderisi, S., Kirkpatrick, B., Bucci, P., Volpe, U., Merlotti, E., Centanaro, F., Catapano, F., Maj, M., 2007. Double dissociation of N1 and P3 abnormalities in deficit and nondeficit schizophrenia. Schizophr. Res. 92, 252-261.

Mulert, C., Pogarell, O., Juckel, G., Rujescu, D., Giegling, I., Rupp, D., et al., 2004. The neural basis of the P300 potential. Eur. Arch. Psychiatry Clin. Neurosci. 254, 190-198.

Nichols, T.E., Holmes, A.P., 2002. Nonparametric permutation tests for functional neuroimaging: a primer with examples. Hum. Brain Mapp. 15, 1-25.

Pae, J.S., Kwon, J.S., Youn, T., Park, H.-J., Kim, M.S., Lee, B., Park, K.S., 2003. LORETA imaging of P300 in schizophrenia with individual MRI and 128-channel EEG. Neuroimage 20, 1552-1560.

Pascual-Marqui, R.D., 2002. Standardized low-resolution brain electromagnetic tomography (sLORETA): technical details. Methods Find. Exp. Clin. Pharmacol. 24, 5-12.

Polich, J., 2007. Updating P300: an integrative theory of P3a and P3b. Clin. Neurophysiol. 118, 2128 2148.

Polich, J., Criado, J.R., 2006. Neuropsychology and neuropharmacology of P3a and P3b. Int. J. Psychophysiol. 60, 172-185.

Polich, J., Herbst, K.L., 2000. P300 as a clinical assay: rationale, evaluation, and findings. Int. J. Psychophysiol. 38, 3-19.

Roach, B.J., Mathalon, D.H., 2008. Event-Related EEG Time-Frequency Analysis: An Overview of Measures and An Analysis of Early Gamma Band Phase Locking in Schizophrenia. Schizophr. Bull. 34, 907-926.

Sabeti, M., Moradi, E., Katebi, S., 2011. Analysis of neural sources of P300 event-related potential in normal and schizophrenic participants, in: Software Tools and Algorithms for Biological Systems. Springer, first ed. Springer, New York.

Sellers, E.W., Donchin, E., 2006. A P300-based brain-computer interface: initial tests by ALS patients. Clin. Neurophysiol. 117, 538-548.

Squires, N.K., Donchin, E., Squires, K.C., 1977. Bisensory stimulation: Inferring decision-related processes from the P300 component. J. Exp. Psychol. Hum. Percept. Perform. 3, 299.

Strobel, A., Debener, S., Sorger, B., Peters, J.C., Kranczioch, C., Hoechstetter, K., Engel, A.K., Brocke, B., Goebel, R., 2008. Novelty and target processing during an auditory novelty oddball: a simultaneous event-related potential and functional magnetic resonance imaging study. Neuroimage 40, 869-883.

Sumiyoshi, T., Higuchi, Y., Itoh, T., Matsui, M., Arai, H., Suzuki, M., Kurachi, M., Sumiyoshi, C., Kawasaki, Y., 2009. Effect of perospirone on P300 electrophysiological activity and social cognition in schizophrenia: a three-dimensional analysis with sloreta. Psychiatry Res. Neuroimaging 172, 180-183.

Sumiyoshi, T., Higuchi, Y., Kawasaki, Y., Matsui, M., Kato, K., Yuuki, H., Arai, H., Kurachi, M., 2006. Electrical brain activity and response to olanzapine in schizophrenia: a study with LORETA images of P300. Prog. Neuropsychopharmacol. Biol. Psychiatry 30, 1299-1303. 
Takahashi, H., Rissling, A.J., Pascual-Marqui, R., Kirihara, K., Pela, M., Sprock, J., Braff, D.L., Light, G.A., 2013. Neural substrates of normal and impaired preattentive sensory discrimination in large cohorts of nonpsychiatric subjects and schizophrenia patients as indexed by MMN and P3a change detection responses. Neuroimage 66, 594-603.

Volpe, U., Mucci, A., Bucci, P., Merlotti, E., Galderisi, S., Maj, M., 2007. The cortical generators of P3a and P3b: a LORETA study. Brain Res. Bull. 73, 220-230.

Wang, J., Hiramatsu, K.-I., Hokama, H., Miyazato, H., Ogura, C., 2003. Abnormalities of auditory P300 cortical current density in patients with schizophrenia using high density recording. Int. J. Psychophysiol. 47, 243-253.

Wang, J., Tang, Y., Li, C., Mecklinger, A., Xiao, Z., Zhang, M., Hirayasu, Y., Hokama, H., Li, H., 2010. Decreased P300 current source density in drug-naive first episode schizophrenics revealed by high density recording. Int. J. Psychophysiol. 75, 249-257.

Wronka, E., Kaiser, J., Coenen, A.M., 2012. Neural generators of the auditory evoked potential components P3a and P3b. Acta Neurobiol. Exp. Wars. 72, 51-64. 


\section{Figure legends}

Fig. 1. Latency and adaptive WOI (aWOI) for each subject. Healthy controls were displayed at left column and Sz patients at right column. A large latency and aWOI variability across subjects can be observed. (a) P300 latency values (in seconds) are represented using a black ' $\mathrm{X}$ ' and aWOI limits are depicted using an arrow. Each arrow represents a particular subject ( $n=38$ for healthy controls and $n=31$ for Sz patients). They were sorted along Y-axis according to P300 response latency. Green arrows represent aWOI limits for the two subjects displayed in the Fig. 1c. (b) P300 aWOI density distribution for each group. The bar plot displays a probability map for each group. It represents the percentage of subjects (in \%) whose adaptive WOI is considered for each post-stimulus time sample. (c) Baseline corrected ERP and 9-samples moving average waveforms at Pz electrode for a healthy control (left) and a Sz patient (right). The P300 and N200 components, as well as fixed WOI (fWOI) and aWOI ranges, are highlighted. In addition, the figure shows the comparison between the length of aWOI and conventional fWOI [250 550] ms poststimulus. (d) sLORETA cortical current density maps are represented for: (i) aWOI; (ii) [250 550] ms fWOI; (iii) [250 350] ms window; (iv) [350 450] ms window; (v) [450 550] ms window. Source activity for fWOI is more distributed over the brain than for aWOI, since sLORETA averaging over a fWOI includes some source information non-related to P300 component. Furthermore, detailed 100 ms-length window analyses show how the source density changes over time.

Fig. 2. Grand-average of normalized cortical current density in healthy controls (left) and Sz patients (right) as a response to distractor (P3a) and target (P3b) tones. In order to reduce inter-subject variability, sLORETA current density at each voxel is normalized by the sum of current density across all voxels. Therefore, the sum of normalized voxel densities will be the unit.

Fig. 3. Three dimensional sLORETA maps of voxel-by-voxel paired sample t-statistics for healthy controls and Sz patients. The cortical current density of P3b and P3a components is compared for each subject. The scale shows negative (blue) and positive (yellow) $t$-values for which alpha is statistically significant after voxel intensity NPT correction for multiple comparisons. An increase of P3b source activity from P3a is represented in yellow and vice versa.

Fig. 4. Differences in brain activation patterns between Sz patients and healthy controls using voxel-byvoxel independent $t$-statistics. The scale shows negative (blue) and positive (yellow) $t$-values for which alpha is statistically significant after cluster size NPT correction for multiple comparisons. Negative tvalues represent larger source activity in control group than in Sz patients and vice versa. 


\section{Tables}

Table 1 Socio-demographic and clinical characteristics of the cohort of subjects enrolled in the study. Values are shown as 'mean \pm standard deviation, SD'. Post-stimulus P300 latency was calculated over a target response using a 9-sample moving average. P3a and P3b amplitudes were obtained from distractor and target responses, respectively. Significance of between-group comparisons is shown in the first column (Kruskal-Wallis test, ${ }^{*} p<0.05$; ${ }^{* *} p<$ 0.001). aWOI adaptive window of interest, CP chronic patients, MTP minimally treated patients, M male, F female, NA not applicable.

\begin{tabular}{ccc}
\hline & Sz patients & Controls \\
\hline Age (years) & $36.25 \pm 9.62$ & $33.35 \pm 12.26$ \\
Gender (M:F) & $21: 10$ & $23: 15$ \\
PANSS-Positive & $19.19 \pm 4.81$ & NA \\
PANSS-Negative & $19.52 \pm 5.69$ & NA \\
PANSS-Total & $73.19 \pm 15.94$ & NA \\
Duration of the illness (months) & $79.73 \pm 103.37$ & NA \\
Number of artifact-free epochs & $88.85 \pm 16.15$ & $84.47 \pm 9.32$ \\
P300 latency at Pz (ms) & $435.23 \pm 67.82$ & $413.05 \pm 72.95$ \\
aWOI length $(\mathrm{ms})$ & $124.26 \pm 61.66$ & $117.47 \pm 47.53$ \\
aWOI lower limit $(\mathrm{ms})$ & $375.48 \pm 68.11$ & $357.79 \pm 66.33$ \\
aWOI upper limit $(\mathrm{ms})$ & $499.74 \pm 85.76$ & $475.26 \pm 91.94$ \\
P3a amplitude at $\mathrm{Pz}(\mu \mathrm{V}){ }^{* *}$ & $0.70 \pm 0.82$ & $1.62 \pm 1.21$ \\
P3b amplitude at $\mathrm{Pz}(\mu \mathrm{V}) *$ & $2.16 \pm 1.18$ & $3.10 \pm 1.47$ \\
\hline
\end{tabular}


Table 2. Results of within-group (P3b vs P3a) analyses. Critical $t$-value $\left(t_{c r i t}\right)$ was estimated for each comparison applying a statistical threshold $(p<0.01)$ to voxel intensity NPT. Gyrus and Brodmann areas (BA) are only displayed when they obtained statistically significant differences after multiple comparisons correction (voxel intensity NPT, $t$ $>t_{\text {crit }}$. Bold names of gyrus and BA represent, respectively, the gyrus and the BA that contain the maximum voxel statistical $t$-value. NS indicates that non-significant differences are found.

\begin{tabular}{|c|c|c|c|c|}
\hline & $\begin{array}{c}p \text {-value } \\
\text { voxel intensity } \\
\text { threshold }\end{array}$ & $\begin{array}{l}\text { Critical } \\
t \text {-value }\end{array}$ & Gyrus & $\begin{array}{c}\text { Brodmann Areas } \\
\text { (BA) }\end{array}$ \\
\hline $\begin{array}{l}\text { Healthy } \\
\text { controls }\end{array}$ & 0.01 & 4.426 & $\begin{array}{l}\text { Paracentral lobule } \\
\text { Postcentral gyrus } \\
\text { Precuneus } \\
\text { Cuneus } \\
\text { Cingulate gyrus } \\
\text { Posterior cingulate }\end{array}$ & $\begin{array}{c}\text { BA3 } \\
\text { BA7 } \\
\text { BA18 } \\
\text { BA19 } \\
\text { BA23 } \\
\text { BA24 } \\
\text { BA30 } \\
\text { BA31 }\end{array}$ \\
\hline $\begin{array}{c}\mathrm{Sz} \\
\text { patients }\end{array}$ & 0.01 & 4.975 & $\begin{array}{c}\text { Medial frontal gyrus } \\
\text { Superior frontal gyrus } \\
\text { Paracentral lobule } \\
\text { Precentral gyrus } \\
\text { Postcentral gyrus } \\
\text { Superior parietal lobule } \\
\text { Precuneus } \\
\text { Sub-gyral } \\
\text { Cingulate gyrus } \\
\text { Posterior cingulate gyrus }\end{array}$ & $\begin{array}{l}\text { BA3 } \\
\text { BA4 } \\
\text { BA5 } \\
\text { BA6 } \\
\text { BA7 } \\
\text { BA8 } \\
\text { BA23 } \\
\text { BA24 } \\
\text { BA31 }\end{array}$ \\
\hline
\end{tabular}


Table 3. Results of between-group brain-source generator analyses. Cluster size NPT were carried out to obtain the critical size of the cluster of voxels and their associated statistical $p_{c o r}$ value. Gyrus and Brodmann areas (BA) are only displayed when they obtained statistically significant differences after multiple comparisons correction (i.e. they formed a greater cluster of voxels than the critical size). Bold names of gyrus and BA represent, respectively, the gyrus and the BA that contain the maximum voxel statistical $t$-value. NS indicates that non-significant differences are found.

\begin{tabular}{|c|c|c|c|c|}
\hline & $\begin{array}{l}p_{\text {cor }} \\
\text { Cluster size }\end{array}$ & $\begin{array}{c}\text { Critical size } \\
\text { (number of } \\
\text { voxels) } \\
\end{array}$ & Gyrus & $\begin{array}{c}\text { Brodmann Areas } \\
(\mathrm{BA})\end{array}$ \\
\hline P3a & 0.038 & 587 & $\begin{array}{c}\text { Superior Frontal Gyrus } \\
\text { Middle Frontal Gyrus } \\
\text { Medial Frontal Gyrus } \\
\text { Cingulate Gyrus }\end{array}$ & $\begin{array}{c}\text { BA6 } \\
\text { BA8 } \\
\text { BA9 } \\
\text { BA24 } \\
\text { BA32 }\end{array}$ \\
\hline $\mathrm{P} 3 \mathrm{~b}$ & 0.033 & 617 & $\begin{array}{c}\text { Medial Frontal Gyrus } \\
\text { Anterior Cingulate } \\
\text { Superior Frontal Gyrus } \\
\text { Rectal Gyrus } \\
\text { Orbital Gyrus } \\
\text { Cingulate Gyrus }\end{array}$ & $\begin{array}{l}\text { BA11 } \\
\text { BA10 } \\
\text { BA25 } \\
\text { BA31 } \\
\text { BA32 } \\
\text { BA33 }\end{array}$ \\
\hline
\end{tabular}




\section{Figures}

Fig. 1
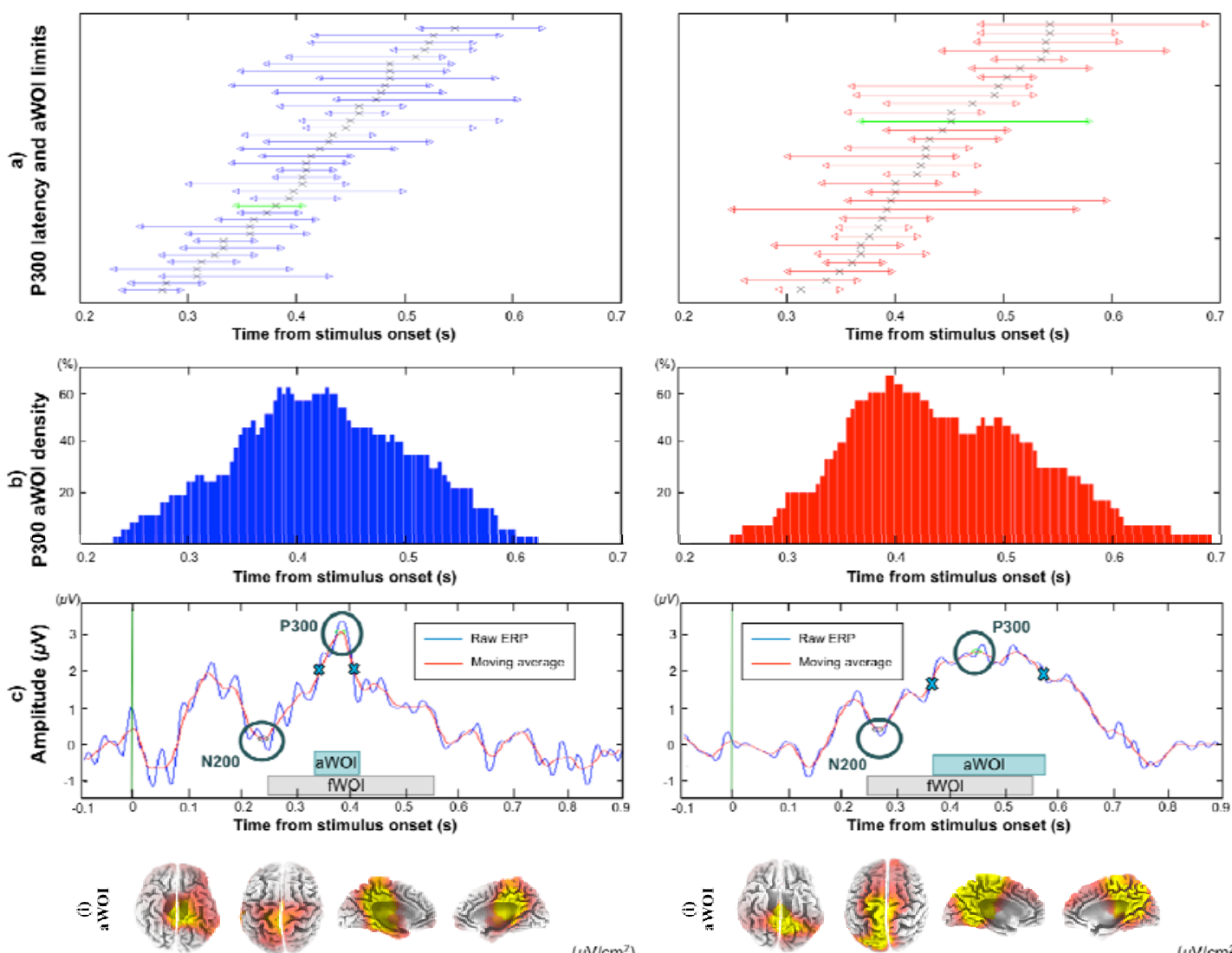

=
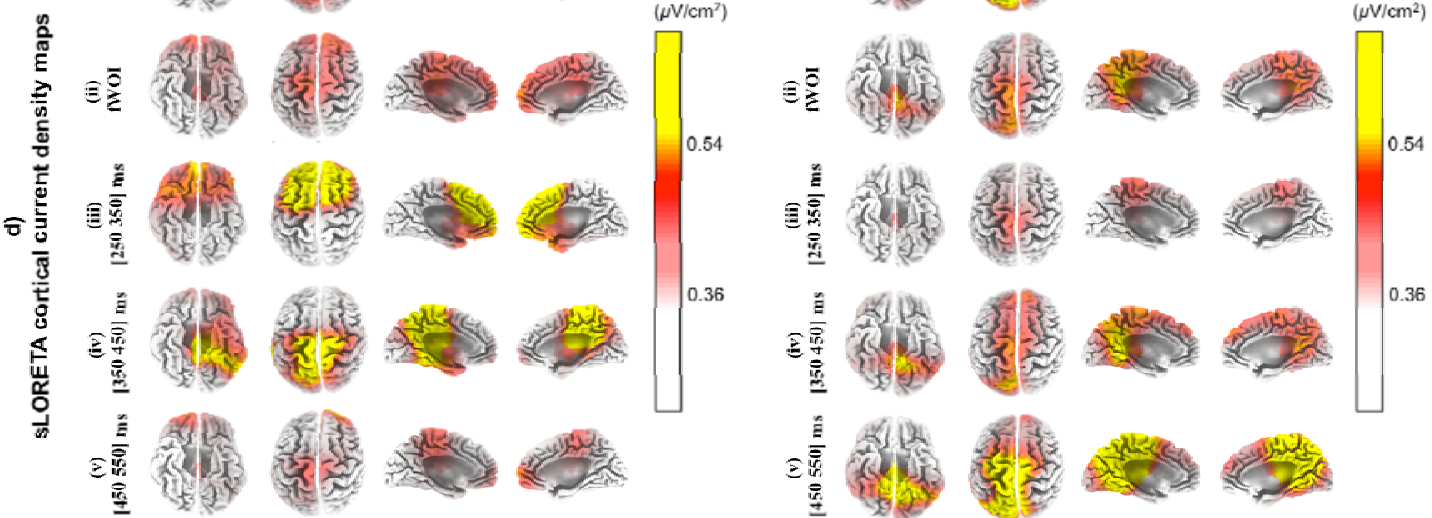

唌 
Fig. 2

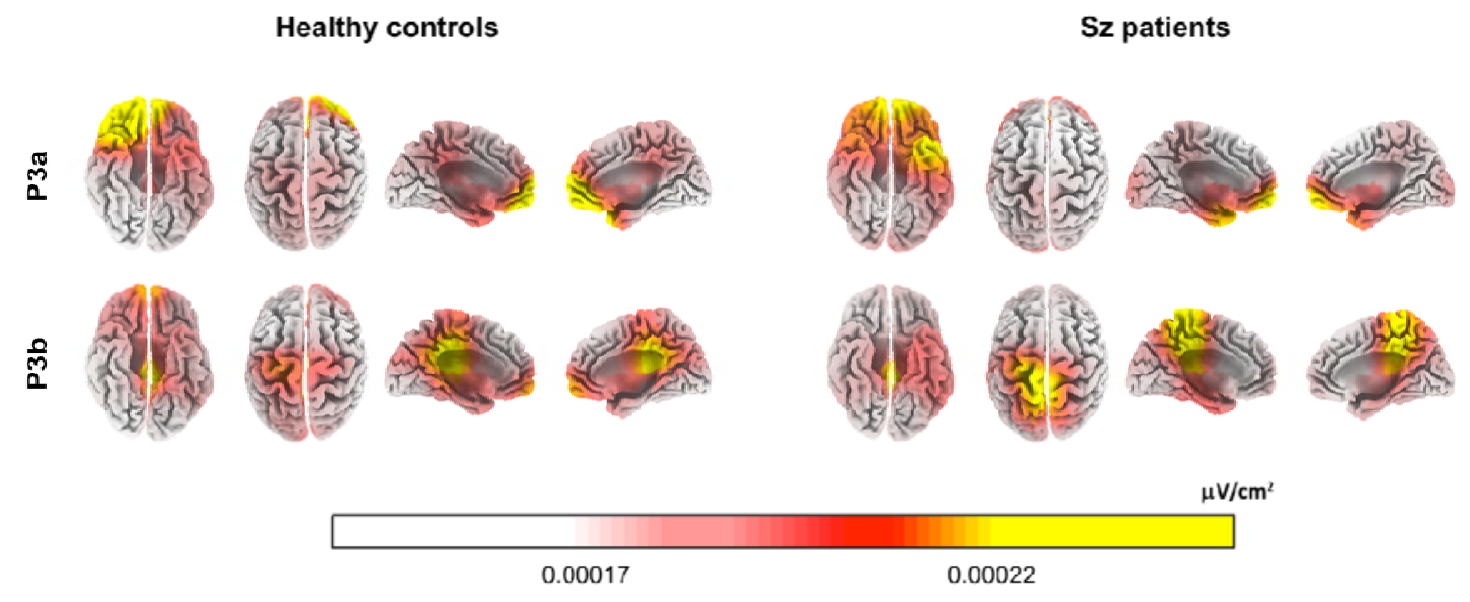

Fig. 3
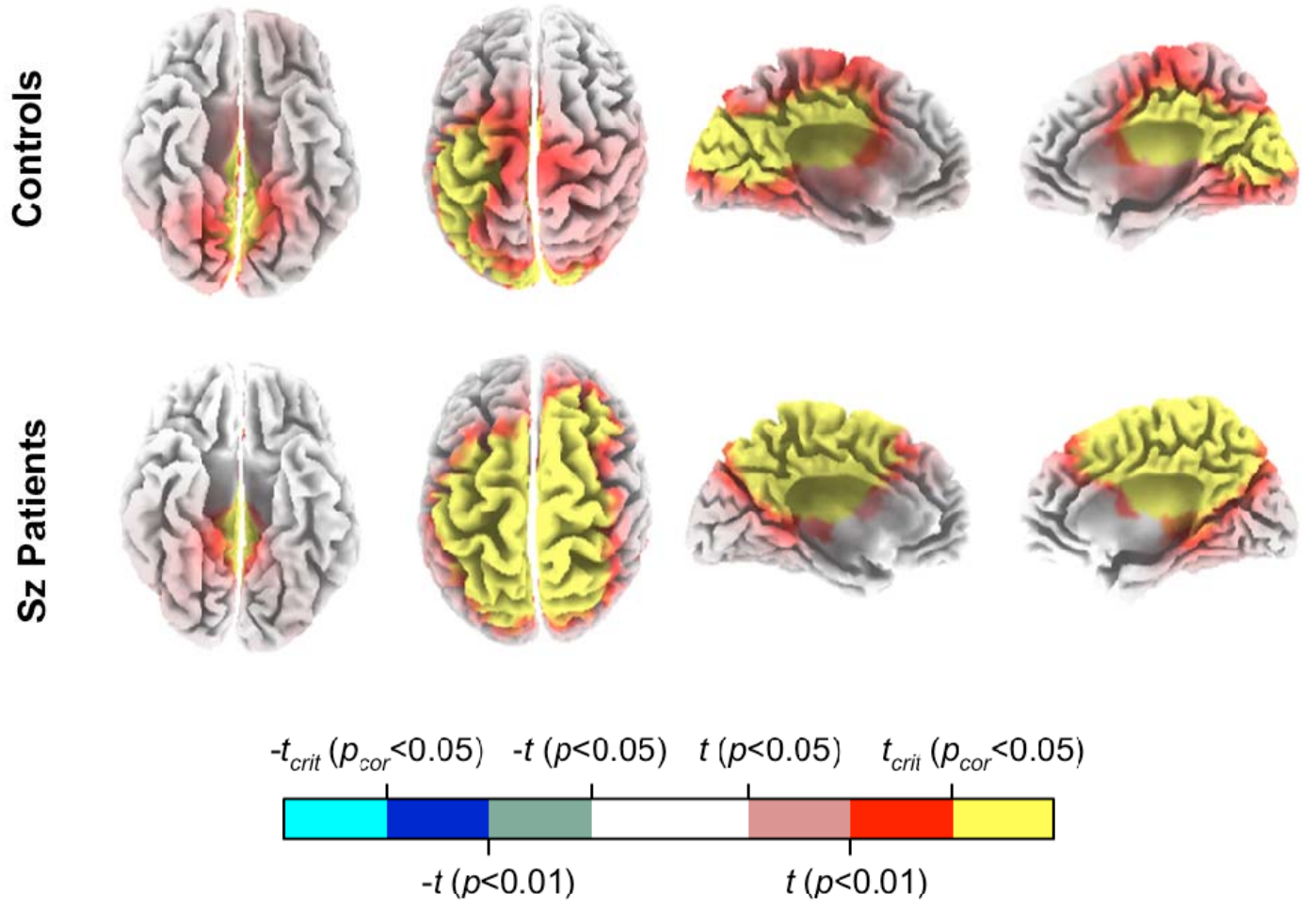
Fig. 4
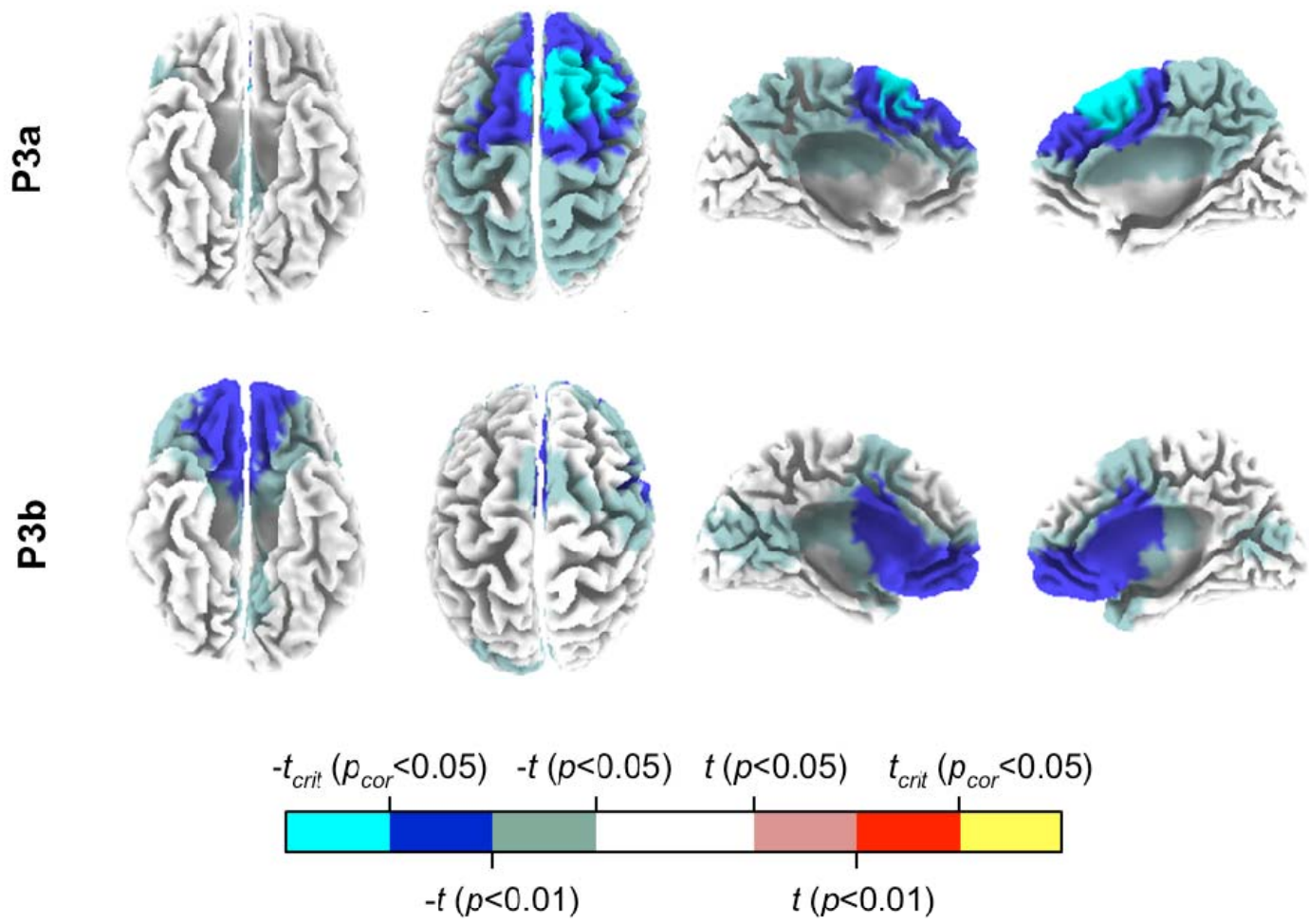